\title{
Papers
}

\section{Cannabis use and mental health in young people: cohort study}

George C Patton, Carolyn Coffey, John B Carlin, Louisa Degenhardt, Michael Lynskey, Wayne Hall

\begin{abstract}
Objective To determine whether cannabis use in adolescence predisposes to higher rates of depression and anxiety in young adulthood.

Design Seven wave cohort study over six years. Setting 44 schools in the Australian state of Victoria. Participants A statewide secondary school sample of 1601 students aged 14-15 followed for seven years.

Main outcome measure Interview measure of depression and anxiety (revised clinical interview schedule) at wave 7 .

Results Some $60 \%$ of participants had used cannabis by the age of $20 ; 7 \%$ were daily users at that point. Daily use in young women was associated with an over fivefold increase in the odds of reporting a state of depression and anxiety after adjustment for intercurrent use of other substances (odds ratio 5.6, $95 \%$ confidence interval 2.6 to 12 ). Weekly or more frequent cannabis use in teenagers predicted an approximately twofold increase in risk for later depression and anxiety $(1.9,1.1$ to 3.3$)$ after adjustment for potential baseline confounders. In contrast, depression and anxiety in teenagers predicted neither later weekly nor daily cannabis use. Conclusions Frequent cannabis use in teenage girls predicts later depression and anxiety, with daily users carrying the highest risk. Given recent increasing levels of cannabis use, measures to reduce frequent and heavy recreational use seem warranted.
\end{abstract}

\section{Introduction}

After increases in cannabis use during the early 1990s, a majority of young people in the United Kingdom, United States, New Zealand, and Australia now use cannabis recreationally. ${ }^{2}$ Despite the high prevalence of cannabis use, uncertainty persists about its physical and psychological consequences. ${ }^{3}$

Among the most prominent concerns have been putative links between use of cannabis and mental disorders. A large intake of cannabis seems able to trigger acute psychotic episodes and may worsen outcomes in established psychosis. ${ }^{45}$ Associations with nonpsychotic disorders have received less attention. Yet evidence for an association between cannabis use and depression and anxiety has grown. ${ }^{6}$ Chronic daily users report high levels of anxiety, depression, fatigue, and their motivation is low. ${ }^{7}$ In one recent survey of young adults, over a third reported symptoms of anxiety that were associated with cannabis use; young women reported these more commonly. ${ }^{8}$ Cross sectional associations between cannabis use and depression and anxiety have now been reported in surveys in both adolescents and adults, ${ }^{910}$ although not all studies have found an association in male participants. ${ }^{11}$

Questions remain about the level of association between cannabis use and depression and anxiety and about the mechanism underpinning the link. Preexisting symptoms might raise the likelihood of cannabis use through a mechanism of self medication. ${ }^{12}$ Alternatively, cannabis use may be more likely in people with a background of social adversity or particular characteristics-factors that might also raise risks for mental disorders. Cannabis may also carry a direct risk for depression and anxiety.

We examined the risks for later depression and anxiety associated with cannabis use in teenagers. Specifically, the study addressed three questions. Firstly, does cannabis use in adolescents predict the development of symptoms of depression and anxiety in young adults? Secondly, do symptoms of depression and anxiety in adolescence predict cannabis use in young adults? Thirdly, is any relation explained by factors such as family background or intercurrent use of other substances?

\section{Methods}

Sample

Between August 1992 and December 1998 we conducted a seven wave cohort study of adolescent health in the Australian state of Victoria. The cohort was defined in a two stage cluster sample, in which we selected two classes at random from each of 44 schools drawn from a stratified frame of government run, Catholic, and independent schools (total number of students 60 905). School retention rates to year nine in the year of sampling were $98 \%$. One class from each school entered the cohort in the latter part of the ninth school year (wave 1) and the second class six months later, early in the 10th school year (wave 2). Participants were subsequently reviewed at six month intervals for the next two years (waves 3 to 6), with a final follow up (wave 7) at the age of 20-21, three years after the final school year in Victoria. In waves 1 to 6 , participants self administered the questionnaire on laptop computers, ${ }^{13}$ and those absent from school were followed up by tele-
Editorial by Rey and
Tennant
Papers pp 1199,
1212

Centre for

Adolescent Health,

Murdoch Children's Research Institute, Parkville, Victoria 3052, Australia

George C Patton

professor of adolescent health

Carolyn Coffey epidemiologist

Clinical

Epidemiology and Biostatistics Unit,

Murdoch Children's

Research Institute

John B Carlin

director of unit

National Drug and

Alcohol Research

Centre, University

of New South

Wales, Sydney 2052,

Australia

Louisa Degenhardt

research fellow

Department of

Psychiatry,

Washington

University School of

Medicine, St Louis,

MO 63110, USA

Michael Lynskey

visiting research

fellow

Office of Public

Policy and Ethics,

Institute for

Molecular

Bioscience,

University of

Queensland,

Brisbane 4072,

Australia

Wayne Hall

professor of bioethics

Correspondence to: G Patton

gpatton@cryptic.

rch.unimelb.edu.au

BMJ 2002;325:1195-8 
phone. The seventh wave of data collection used computer assisted telephone interviews. All stages of the study were approved by the ethics committee of the Royal Children's Hospital.

From a total sample of 2032 students, 1947 (95.8\%) participated at least once during the first six (adolescent) waves. In wave 7, 1601 young adults (79\% of the initial sample or $82 \%$ of teenage participants) were interviewed between April and December 1998. Response rates are shown in figure 1 . Reasons for noncompletion at follow up were refusal $(n=152)$, loss of contact $(n=192)$, and death $(n=2)$. We examined characteristics of non-completers in a logistic regression model. Male participants were overrepresented (odds ratio 1.9, 95\% confidence interval 1.5 to 2.4 ), as were parental divorce or separation (1.8, 1.4 to 2.5), and daily tobacco smoking at study inception (2.1, 1.5 to 2.9$)$. Neither teenage depression and anxiety nor cannabis use were independently associated with loss to follow up. The mean age at wave 1 was 14.5 (SD 0.5) years; at wave 7 it was $20.7(0.5)$ years. Of the 1601 participants in wave 7, $1130(71 \%)$ still lived at home, $429(27 \%)$ lived with others, and 42 (3\%) lived alone. A total of 1345 (82\%) had completed the final year of school; $1355(85 \%)$ had started post-school study.

\section{Measures}

We used the computerised revised clinical interview schedule (CIS-R) to assess depression and anxiety at each wave. ${ }^{14}$ The schedule provides data on the frequency, severity, persistence, and intrusiveness of 14 common psychiatric symptoms and has been widely used in population based surveys. ${ }^{15}$ A total score of 12 or greater was taken to define a mixed state of depression and anxiety at a lower threshold than syndromes of major depression and anxiety disorder but one where clinical intervention would still be appropriate. ${ }^{16}$

We assessed cannabis use on the basis of self reported frequency of use in the previous six months in waves 1 to 6 and in the previous 12 months in wave 7 . This allowed classification as never used, less than weekly use, at least weekly use, and daily use (defined as using on five or more days per week), and initiation after wave 6 .

We assessed use of alcohol, tobacco, and other illicit drugs (including ecstasy, heroin, amphetamines, LSD, and steroids) on the basis of self reported frequency of use and with retrospective diaries over seven days for participants reporting recent drinking or smoking. Participants drinking on three or more days in the previous week were classified as frequent drinkers.

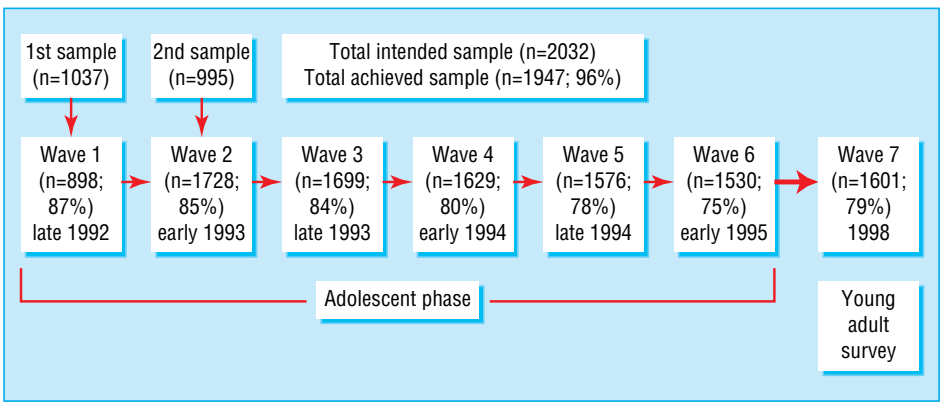

Fig 1 Participation rates of 2032 secondary school students in the Victorian adolescent health cohort study. The percentages in waves 2-7 are the proportions of the total intended sample for which complete data were collected
We assessed antisocial behaviour in waves 1 to 6 by using items from the self reported early delinquency scale that covered property damage, interpersonal violence, and theft. ${ }^{17}$

\section{Analysis}

We collected data at a developmental point when young people are difficult to trace because of high mobility. Although the response rate was high and attrition low, $70 \%$ of respondents missed at least one wave of data collection, which led to potential bias in summary measures of exposure to cannabis and mental health problems calculated from the six waves of data collection among adolescents. To circumvent this, we used multiple imputation with five complete datasets created by imputation under the multivariate mixed effects model of Schafer and Yucel, incorporating the covariates sex, age, rural or urban residence, and parental education (available for all participants). ${ }^{18} 19$ These covariates were strongly associated with missingness, and the model incorporated a random effects structure to accommodate correlation within participants over time. We constructed principal measures by classifying participants according to whether they fell into categories of interest at least once during wave 1 to 6 (adolescence) and, separately, in wave 7 (young adulthood). Data analysis was performed with Stata 7 . We modelled associations by univariate and multivariate logistic regression analyses and used Wald tests and related confidence intervals to assess statistical significance and precision.

\section{Results}

Altogether 71 male participants $(9.7 \%, 95 \%$ confidence interval $7.5 \%$ to $12 \%)$ and $188(22 \%, 19 \%$ to $25 \%)$ of female participants reported depression and anxiety as young adults (odds ratio 2.6, 1.9 to 3.5). Sixty six per cent $(484 / 731)$ of male participants and 52\% $(448 / 859)$ of female participants reported using cannabis at some time (11 participants did not respond to this question), with three quarters starting use when they were teenagers. Twenty per cent $(146 ; 17 \%$ to $22 \%)$ of male participants and $8 \%(69 ; 6 \%$ to $10 \%)$ of female participants were using cannabis at least weekly, with $10 \%(73 ; 8 \%$ to $12 \%)$ of young men and $4 \%(37$; $3 \%$ to $6 \%$ ) of young women using it daily.

\section{Cannabis and depression in young adults}

The prevalence of depression and anxiety increased with higher extents of cannabis use, but this pattern was clearest in female participants (table 1). We used logistic regression to analyse the level of association between depression and anxiety and cannabis use in young adults (table 2) after adjustment for concurrent substance use. We found a significant interaction between sex and daily cannabis use. In the adjusted model, young women who used cannabis daily had an over fivefold increase in the odds of depression and anxiety found in non-users.

\section{Cannabis in adolescence and depression in young} adults

We used logistic regression to examine the prediction of depression and anxiety in young adults by cannabis use in adolescence. In the univariate analysis a dose response was evident: daily use in female teenagers 
Table 1 Prevalence of depression and anxiety according to cannabis use by sex in 1590 young adults in wave 7 ( $n=1601$ ) of the Victorian adolescent health cohort study

\begin{tabular}{|c|c|c|c|c|c|c|}
\hline \multirow{2}{*}{$\begin{array}{l}\text { Frequency of cannabis use in } \\
\text { previous } 12 \text { months }\end{array}$} & \multicolumn{3}{|c|}{ Men } & \multicolumn{3}{|c|}{ Women } \\
\hline & No & $\%(95 \% \mathrm{CI})$ & Odds ratio* $(95 \% \mathrm{Cl})$ & No & $\%(95 \% \mathrm{Cl})$ & Odds ratio* $(95 \% \mathrm{CI})$ \\
\hline None to $<5$ times ever & 523 & $9(6$ to 11$)$ & (1) & 744 & 19 (17 to 22$)$ & (1) \\
\hline 5 times ever to less than weekly & 62 & 10 (2 to 17$)$ & $1.1(0.46$ to 2.8$)$ & 46 & 17 (6 to 29$)$ & $0.87(0.40$ to 1.9$)$ \\
\hline 1 to 4 times per week & 73 & 12 (5 to 20$)$ & $1.5(0.70$ to 3.2$)$ & 32 & $31(14$ to 48$)$ & $1.9(0.87$ to 4.1$)$ \\
\hline Daily & 73 & 15 (7 to 23$)$ & $1.9(0.93$ to 3.8$)$ & 37 & 68 (52 to 83 ) & 8.6 (4.2 to 18$)$ \\
\hline
\end{tabular}

*Obtained from univariate logistic regression models.

11 (7 female) participants in wave 7 did not answer the questions about cannabis use.

*Obtained from univariate logistic regression models.

predicted fourfold higher odds of later depression and anxiety (odds ratio 4.2, 1.6 to 11 ), weekly use a twofold elevation $(2.3,1.3$ to 4.2$)$. In the multivariate model we collapsed the top categories of cannabis use (table 3). The interaction between sex and weekly or more frequent use was significant. An almost twofold increase in risk for weekly or more frequent users who were female persisted after adjustment for potential confounders.

\section{Depression in adolescence and cannabis in young adults}

We considered whether depression and anxiety in adolescence predicted later cannabis use in young adulthood in two further logistic regression models, examining the predictions of weekly and daily use (table 4). After adjustment for adolescent cannabis use and other potential confounders, adolescent depression and anxiety predicted neither weekly nor daily use.

\section{Discussion}

Around $60 \%$ of the statewide secondary school sample had used cannabis recreationally by young adulthood; most participants first experimented while at secondary school. By young adulthood $7 \%$ were daily users and in young women this level of use was associated with over five times the odds of depression and anxiety found in non-users. In young women, weekly use as teenagers predicted a twofold increase in later depression and anxiety and daily use a fourfold increase. In contrast, depression in teenagers did not predict higher cannabis use.

\section{Strengths}

Earlier cohort studies had a limited capacity to address the key questions of this study. One study reported a prospective relation between cannabis use and later depression but started well after the risk period of onset for both. ${ }^{20}$ Two important studies in adolescence examined either monthly cannabis use or use in the preceding year-doses that in the light of this study are unlikely to be associated with mental health problems. ${ }^{21} 22$

Our close to representative sample, high rates of participation, and frequent measures during participants' teenage years are strengths of this study. A telephone interview strategy was used in data collection in the last wave, and, although prevalence estimates may vary slightly as a result, it is unlikely to have caused a systematic bias in patterns of association. The use of multiple imputation minimised measurement biases arising from missing data during the teenage years, but we did not attempt to adjust for differential participation of young adults. Even though depression and anxiety in teenagers and cannabis use did not predict dropout from the study, the difference in non-responders on other factors (for example, sex or family structure) may have had some bearing on the specification of associations.

\section{What the results might mean}

Possible explanations for the high degree of depression and anxiety found in young women who used cannabis often include underlying characteristics that predispose to both anxiety and depression, self medication of pre-existing depressive symptoms, and an adverse effect of cannabis on mental health. ${ }^{21}$ The association with cannabis use persisted after adjustment for concurrent use of alcohol, tobacco, and other illicit substances as well as indices of family disadvantage-findings consistent with a more direct relation. We considered self medication with cannabis

Table 2 Association between cannabis use in the previous 12 months and depression and anxiety in 1590 young adults in wave $7(n=1601)$ of the Victorian adolescent health study, derived from a multivariate logistic model

\begin{tabular}{lcc} 
Cannabis use & No & Adjusted odds ratio (95\% CI) \\
\hline None to $<5$ times in previous 12 months & 1267 & 1 \\
\hline 5 times ever to <weekly & 108 & $0.80(0.44$ to 1.5$)$ \\
\hline $1-4$ times/week & 105 & $1.1(0.60$ to 2.0$)$ \\
\hline Daily $:$ & & \\
\hline Men & 73 & $1.1(0.55$ to 2.6$)$ \\
\hline Women & 37 & $5.6(2.6$ to 12$)$ \\
\hline Female sex in the absence of daily cannabis use & 822 & $2.5(1.8$ to 3.4$)$ \\
\hline
\end{tabular}

Odds ratios are adjusted for parental separation, parental education, current smoking, frequency of drinking, and use of other illicit drugs.

11 (7 female) participants did not answer the questions about cannabis use in wave 7.

*Wald test for interaction between daily cannabis use and sex: $\mathrm{P}=0.003$.

Table 3 Association of cannabis use in teenagers with later depression and anxiety in 1601 young adults in wave 7 of the Victorian adolescent health cohort study

\begin{tabular}{|c|c|c|c|}
\hline Measures in waves 1-6 & $\mathrm{No}^{*}$ & $\begin{array}{l}\text { Unadjusted odds ratio } \\
(95 \% \mathrm{CI})\end{array}$ & $\begin{array}{c}\text { Adjusted odds ratio } \\
(95 \% \mathrm{Cl}) \dagger\end{array}$ \\
\hline Depression and anxiety (at least one wave) & 744 & $6(4.3$ to 8.4$)$ & 5.1 (3.6 to 7.3$)$ \\
\hline \multicolumn{4}{|l|}{ Maximal cannabis use } \\
\hline None & 1083 & 1 & 1 \\
\hline$<$ Weekly & 332 & $1.5(1.1$ to 2.1$)$ & 1.4 (94 to 2.0$)$ \\
\hline \multicolumn{4}{|l|}{ >Weeklył: } \\
\hline Male teenagers & 108 & $0.62(0.24$ to 1.6$)$ & $0.47(0.17$ to 1.3$)$ \\
\hline Female teenagers & 78 & $2.6(1.6$ to 4.3$)$ & $1.9(1.1$ to 3.3$)$ \\
\hline $\begin{array}{l}\text { Female sex in the absence of }>\text { weekly } \\
\text { cannabis use }\end{array}$ & 788 & $2.3(1.6$ to 3.1$)$ & $1.6(1.1$ to 2.3$)$ \\
\hline
\end{tabular}

*Numbers for adolescent cannabis use and depression and anxiety were estimated from five imputed datasets.

†Odds ratios by the highest frequency of cannabis use in teenagers (waves 1 to 6 ), obtained by using a multivariate logistic model, adjusted for teenagers' depression and anxiety, alcohol use, antisocial behaviour, parental separation, and parental education.

$\ddagger$ Wald test for interaction between more frequent than weekly cannabis use and sex: unadjusted $P<0.001$, adjusted $\mathrm{P}=0.011$. 
Table 4 Association of cannabis use in teenagers (waves 1-6) with later depression and anxiety in 1590 young adults in wave $7(n=1601)$ of the Victorian adolescent health cohort study

\begin{tabular}{lccc} 
& & \multicolumn{2}{c}{ Odds ratio (95\% Cl) $\dagger$} \\
\cline { 3 - 4 } Measures in waves $\mathbf{1}$ to $\mathbf{6}$ & $\mathbf{N o}^{*}$ & >Weekly use & Daily use \\
\hline Depression and anxiety: at least one wave & 739 & $1.2(0.86$ to 1.8$)$ & $1.3(0.80$ to 2.2$)$ \\
\hline Maximal cannabis use: & & & \\
\hline None & 1074 & 1 & 1 \\
\hline <Weekly & 330 & $3.7(2.4$ to 5.6$)$ & $3.1(1.7$ to 5.7$)$ \\
\hline$>$ Weekly & 185 & $15(9.2$ to 23$)$ & $15(8.2$ to 27$)$ \\
\hline Female sex & 859 & $0.38(0.26$ to 0.54$)$ & $0.5(0.29$ to 0.77$)$ \\
\hline
\end{tabular}

11 (7 female) participants in wave 7 did not answer the questions about cannabis use.

${ }^{*}$ Numbers for adolescent cannabis use and depression and anxiety estimated from five imputed datasets.

†Odds ratios obtained by using multivariate logistic models, adjusted for teenagers' cannabis use, drinking frequency, parental separation, and parental education.

but found no prospective relation between depression and anxiety in adolescence and later frequent cannabis use, consistent with an earlier report. ${ }^{22}$

The persistence of associations in the multivariate models and the evidence for a prospective doseresponse relation are consistent with a view that frequent use of cannabis in young people increases the risks of later depression and anxiety. Psychosocial mechanisms-for example, the adoption of a countercultural lifestyle-possibly underlie the association. Social consequences of frequent use include educational failure, dropout, unemployment, and crimeall factors that may lead to higher rates of mental disorders. Because risks seem confined largely to daily users, however, the question about a direct pharmacological effect remains. Cannabinoid receptors (CB1) are found widely in the central nervous system, with a distribution that is consistent with effects on a wide range of brain functions including memory, emotion, cognition, and movement. ${ }^{23}$

Cannabis use in young people remains a controversial area, and absence of good data has handicapped the development of rational public health policies. ${ }^{3}$ These findings contribute to evidence that frequent cannabis use may have a deleterious effect on mental health beyond a risk for psychotic symptoms. Strategies to reduce frequent use of cannabis might reduce the level of mental disorders in young people.

Contributors: GCP was the principal investigator and prepared the manuscript. CC was the study coordinator and contributed to data analysis and manuscript preparation. JBC contributed to the data analysis and manuscript preparation. LD, ML, and WH contributed to the preparation of the manuscript. GCP is the guarantor.

Funding: National Health and Medical Research Council and Victorian Health Promotion Foundation.

Competing interests: None declared.

1 Smart RG, Ogborne AC. Drug use and drinking among students in 36 countries. Addict Behav 2000;25:455-60.

2 Ramsay, M, Spiller J. Drug use declared in 1996: latest results from the British crime survey. London: Home Office, 1977.

3 Strang J, Witton J, Hall W. Improving the quality of the cannabis debate: defining the different domains. BMJ 2000;320:108-10.

4 Linszen DH, Dingemans PM, Lenior ME. Cannabis abuse and the course of recent-onset schizophrenic disorders. Arch Gen Psychiatry 1994;51:273-9.

5 Hall W. Cannabis use and psychosis. Alcohol Rev 1998;17:433-44.

6 Degenhardt L, Hall W, Lynskey MT. Alcohol, cannabis and tobacco use among Australians: a comparison of their associations with other drug use and use disorders, affective and anxiety disorders, and psychosis. Addiction 2001;96:1603-14

7 Reilly D, Didcott R, Swift W. Long-term cannabis use: characteristics of users in Australian rural areas. Addiction 1998;93:837-46.

8 Thomas H. A community survey of adverse effects of cannabis use. Drug Alcohol Depend 1996;42:201-7.

9 Rey JM, Sawyer MG, Raphael B, Patton GC, Lynskey MT. The mental health of teenagers who use marijuana. Br J Psychiatry 2001;180:216-21.

\section{What is already known on this topic}

Frequent recreational use of cannabis has been linked to high rates of depression and anxiety in cross sectional surveys and studies of long term users

Why cannabis users have higher rates of depression and anxiety is uncertain

Previous longitudinal studies of cannabis use in youth have not analysed associations with frequent cannabis use

\section{What this study adds}

A strong association between daily use of cannabis and depression and anxiety in young women persists after adjustment for intercurrent use of other substances

Frequent cannabis use in teenage girls predicts later higher rates of depression and anxiety

Depression and anxiety in teenagers do not predict later cannabis use; self medication is therefore unlikely to be the reason for the association

10 Troisi A, Pasini A, Saracco M. Psychiatric symptoms in male cannabis users not using other illicit drugs. Addiction 1998;93:487-92

11 Green BE, Ritter C. Marijuana use and depression. J Health Soc Behav 2000;41:40-9.

12 Paton S, Kessler R, Kandel D. Depressive mood and adolescent illicit drug use: a longitudinal analysis. J Gen Psychol 1977;92:267-87.

13 Paperny DM, Aono JY, Lehman RM. Computer assisted detection and intervention in adolescent high-risk health behaviour. I Pediatr 1990;116:456-62.

14 Lewis G, Pelosi AJ. The manual of CIS-R. London: Institute of Psychiatry, 1992.

15 Bebbington PE, Dunn G, Jenkins R, Lewis G, Brugha TS, Farrell M, et al. The influence of age and sex on the prevalence of depressive conditions: report from the national survey of psychiatric morbidity. Psychol Med 1998;28:9-19.

16 Lewis G, Pelosi AJ, Araya R, Dunn G. Measuring psychiatric disorder in the community: a standardized assessment for use by lay interviewers. Psychol Med 1992;22:465-86.

17 Moffitt TE, Silva PA. Self-reported delinquency: results from an instrument for New Zealand. Aust N Z J Criminol 1988;21:227-40.

18 Rubin DB. Multiple imputation for non-response in surveys. New York: Wiley, 1987.

19 Schafer JL, Yucel RM. Computational strategies for multivariate linear mixed-effects models with missing values. I Comput Graph Stat 2002;11:437-57

20 Bovasso GB. Cannabis abuse as a risk factor for depressive symptoms. Am J Psychiatry 2001;158:2033-7.

21 Fergusson DM, Horwood LJ. Early onset cannabis use and psychosocial adjustment in young adults. Addiction 1997;92:279-96.

22 McGee R, Williams S, Poulton RG, Moffitt TE. A longitudinal study of cannabis use and mental health from adolescence to early adulthood. Addiction 2000;95:491-503.

23 Ameri A. The effects of cannabinoids on the brain. Prog Neurobiol 1999;58:315-48.

(Accepted 15 August 2002)

\section{Endpiece}

\section{Surgical innovation}

It is infinitely better to transplant a heart than to bury it so it can be devoured by worms.

Christiaan Barnard (1922-2001), who performed the first human heart transplant in 1967

Submitted by Max Edwards,

surgical trainee, London 